\title{
5 Orthopädische Aspekte bei Kindern und Erwachsenen
}

\author{
Falk Thielemann, Klaus-Peter Günther und Maik Stiehler
}

Übergewichtige Patienten klagen häufig über Schmerzen des Haltungs- und Bewegungsapparates. Neben funktionellen Behinderungen durch die Weichteilzunahme am Rumpf und an den Extremitäten sind meist Fehlstellungen und strukturelle Veränderungen an den lasttragenden Gelenken der unteren Extremitäten und der Wirbelsäule für die beklagten Beschwerden verantwortlich.

Bereits im Kindes- und Jugendalter bestehen klar belegte Zusammenhänge zwischen der Entwicklung pathologischer Fuß-, Beinachsen- und Hüftfehlstellungen und frühzeitig manifestem Übergewicht oder gar Adipositas. Unfallstatistiken belegen die erhöhte Verletzungsgefahr übergewichtiger Kinder und Jugendlicher im Alltag und bei sportlichen Aktivitäten.

Die schnelle Verfügbarkeit von Nahrungsmitteln in den Industriestaaten mit der Steigerung der kohlehydratreichen Ernährung erhöht das Risiko der Erkrankung an Diabetis mellitus und seinen Folgen. Die Versorgung des diabetischen Fußsyndroms stellt bei Erwachsenen eine wachsende interdisziplinäre Herausforderung dar. Übergewicht gilt als wesentlicher Risikofaktor für pathologische Veränderungen der Gelenkbiomechanik, für Fehlbelastungen und die Manifestation von Arthrosen großer lasttragender Gelenke. Der Einfluss von Adipositas auf das klinisch-funktionelle Ergebnis nach endoprothetischer Versorgung wird kontrovers diskutiert. Der optimalen Behandlung der Adipositas in allen Lebensabschnitten zur Primär- und Sekundärprävention der im Folgenden dargestellten Erkrankungen und der aus ihnen resultierenden Folgeschäden am Bewegungsapparat kommt damit eine zentrale Bedeutung zu.

\subsection{Fußdeformitäten}

Grundsätzlich ist eine milde Knick-Senk-Fußstellung mit geringfügig abgeflachtem Längsgewölbe im Kleinkindalter ein physiologischer Befund und im Erwachsenenalter ohne wesentlichen pathologischen Wert. Die milde Deformität kann bis in das hohe Alter beim Stehen und Gehen durch die invertorisch wirkenden Muskeln aktiv kompensiert werden. Dieser aktiv muskulären und passiv ligamentären Kompensationsfähigkeit sind Grenzen gesetzt.

Die frühzeitige Manifestation bzw. langfristige Exposition von Übergewicht stellt neben einer konstitutionellen Bandschwäche ein wesentliches Bindeglied für eine mögliche Dekompensation der subund prätalaren Fußgewölbestruktur und damit der dynamischen Stoßdämpferfunktion der Füße dar.

In Fällen einer ausgeprägten Instabilität des tarsalen Gelenkkomplexes kommt es unter Belastung zur Kippung des Talus über den Kalkaneus nach medial und kaudal. Der Kalkaneus evertiert dabei in eine Valgusstellung und die Wadenmuskulatur mit der Achillessehne verkürzen sich. Obwohl keine universal akzeptierte Definition für einen Plattfuß existiert, sind folgende klinische und anatomische Charakteristika während der Standphase typische Merkmale dieser Deformität:

nückfußvalgus = ausgeprägte Eversion des subtalaren Komplexes 
- Prominenz des Taluskopfes an der medioplantaren Belastungsfläche $=$ medioplantare Subluxation des Talus im Talokalkaneargelenk

- Vorfußabduktion = Dorsalflexion und Abduktion des Os naviculare und Os cuboideum (Chopart'sche Gelenkreihe)

- supinierter Vorfuß in Relation zur evertierten Rückfußstellung

Von den betroffenen Kindern und Erwachsenen werden neben der fehlenden alltäglichen Belastungsfähigkeit diffuse Beschwerden in den Füßen aber häufig auch im gesamten muskuloskelettalen System beklagt.

Der klinische Untersuchungsbefund zeigt neben den oben genannten Merkmalen eine unterschiedlich ausgeprägte Verkleinerung oder gar fehlende Aussparung der Belastungsfläche in der Mitte des medialen Fußrandes, d.h. einen Verlust des Fußlängsgewölbes (s. Abb. 8). In ausgeprägten Fällen kann sogar der Kontaktverlust der lateralen Säule mit kompletter Verlagerung der plantaren Belastungsfläche nach medial resultieren. Häufig bestehen in diesen Fällen medioplantare Beschwielungen in Höhe des luxiert stehenden Taluskopfes oder gar Hautulcera als Ausdruck des pathologischen plantaren Belastungsmusters.

\subsubsection{Wissenschaftliche Daten zum Einfluss von Übergewicht auf den wachsenden Fuß}

Auf die erhöhte klinische Prävalenz von Plattfüßen bei adipösen Kindern (BMI > 30) wurde von Horn (2002) in einer Studie aus der Universität Ulm hingewiesen. Sie legt den Zusammenhang zwischen kindlichem Übergewicht und dem Auftreten einer Plattfußdeformität nahe. In einer Reihe von wissenschaftlichen Untersuchungen wurde versucht, den Einfluss von Übergewicht und Adipositas auf das wachsende Fußskelett näher zu untersuchen: Riddiford-Harland et al. (2000) konnten beispielsweise anhand der statischen Fußabdrücke von 431 präpubertären australischen Kindern signifikante Unterschiede im plantaren Belastungsmuster zwischen übergewichtigen und nicht übergewichtigen Kindern nachweisen. Dowling et al. (2001; 2004) veröffentlichten verschiedene Untersuchungen zur Erfassung des Effektes von Übergewicht auf das plantare Druckverteilungsmuster bei präpubertären Kindern. Ausgewertet wurden statische und dynamische Fußdruckmessungen von Kindern mit manifestem Übergewicht (BMI 25,8 $\pm 3,8$ ) im Vergleich zu Kindern

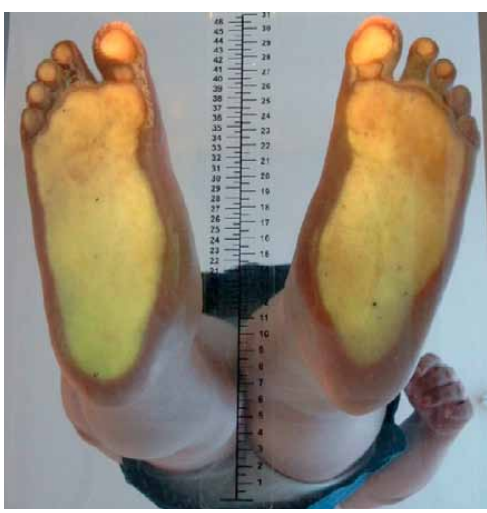

Abb. 8 Podogramm eines übergewichtigen 12-jährigen Jungen mit flexiblen Plattfüßen beidseits (BMI 27,3). Die plantare Belastungsfläche ist komplett nach medial verschoben. Die Aussparung der Belastung am medialen Fußrand, d.h. das Längsgewölbe beider Füße, existiert nicht.

einer gleichaltrigen nichtübergewichtigen Kontrollgruppe (BMI 16,8 \pm 2 ). Die Berechnungen der Kraftund Druckverteilung im be- und entlasteten Zustand deckten interessante Unterschiede des Gangverhaltens und der statischen und dynamischen Vor- und Rückfußbelastung bei übergewichtigen Kindern gegenüber der nicht übergewichtigen Kontrollgruppe auf. Die Autoren postulieren, dass Dyskomfort und/oder strukturelle Pathologien mit einem erhöhten plantaren Vorfußdruckverteilungsmuster assoziiert sind und mittelfristig zu einer Absenkung des medialen Fußrandes beim Gehen führen.

\subsubsection{Wissenschaftliche Daten zur klinischen Relevanz der kindlichen Plattfüße für Erwachsene}

Übergewicht und Adipositas sind, abhängig von der Expositionsdauer, wichtige potenzielle Risikofaktoren für die frühe Dekompensation tarsaler Gelenkstrukturen. Untersuchungen zum natürlichen Verlauf der Plattfußdeformität und daraus resultierender Fußbeschwerden im Erwachsenenalter sind rar. Bruckner und Rosler publizierten 1981 eine Querschnittstudie zu Fußbeschwerden und orthopädisch relevanten Veränderungen in einem Kollektiv von 103 Frauen. Sie unterstreichen den Zusammenhang zwischen dem Auftreten schmerzhafter Fußdeformitäten und bestehender Adipositas. Insbesondere hinsichtlich der Entwicklung einer Vorfußdeformität mit Verlust des Quergewölbes und der Ausbildung 
eines Hallux valgus konnte eine Korrelation mit erhöhtem Körpergewicht herausgearbeitet werden. Mertz und Mertz (1982) beschreiben die Adipositas als ein wichtiges Bindeglied zwischen Hyperurikämie oder Gicht und einer Croßzehengrundgelenkarthrose (Hallux rigidus). Sie postulieren die Akzeleration der Genese dieser speziellen Arthroseform durch Adipositas.

\subsubsection{Das diabetische Fußsyndrom}

Übergewicht und Adipositas sind häufig die Folge einer kohlehydratreichen Ernährung. Damit steigt insbesondere das Risiko einer Diabetes Typ 2 Erkrankung. Je nach zeitlicher Länge eines schlecht eingestellten Diabetes mellitus nimmt die Gefahr diabetesassoziierter Fußprobleme zu. Für den diabetischen Fuß sind folgende Veränderungen von pathologischer Bedeutung:

- diabetische Makroangiopathie

- diabetische Mikroangiopathie

- diabetische Neuropathie

- diabetische Osteoarthropathie

- diabetische Fettgewebsatrophie

- diabetische Myatrophie

Veränderungen der Gefühlsempfindung der Füße, Störungen der Schweißsekretion und Durchblutung führen zu Schwielen, Druckulzera, Infektion mit Bakterien und Pilzen mit chronischen Weichteilund Knocheninfekten bis hin zur diabetischen Gangrän. Epidemiologische Daten lassen den Schluss zu, dass etwa 40-75\% der nicht-traumatischen Amputationen heute auf diabetesassoziierte Erkrankungen zurückführen sind.

Die Betreuung dieser sehr schwierigen Patientengruppe kann aus diesem Grund nur im Rahmen eines eng abgestimmten interdisziplinären Teamkonzeptes erfolgreich sein. Die Integration der Patienten in einer gut organisierten Fußambulanz ist wünschenswert. Neben der optimalen internistischen Einstellung der Stoffwechselentgleisungen benötigen die Patienten eine spezialisierte Betreuung bei der Fußpflege, der Schuhversorgung, eine diätetische Beratung und in Abhängigkeit der Risikogruppe die Anbindung an spezialisierte operative Fachrichtungen.

Sowohl bei der physiologischen Aufrichtung des Längsgewölbes am kindlichen Knick-Senk-Fuß als auch bei der Entstehung adulter Fehlstellungen und Gelenk- arthrosen im Mittel und Vorfuß kann das Vorliegen von Übergewicht einen negativen Einfluss ausüben.

Übergewicht und Adipositas sind häufig mit Stoffwechselentgleisungen verbunden. Der Diabetes mellitus und seine Folgen stellen eine besondere Herausforderung an die enge interdisziplinäre Zusammenarbeit bei der Betreuung von Patienten mit diabetischem Fußsyndrom.

\subsection{Deformitäten der Beinachsen}

\subsubsection{Physiologische Entwicklung der Beinachsen und ihre Belastung}

Während der Wachstumsphase vermögen die gesunden Wachstumsfugen der großen Röhrenknochen Biegebelastung, d.h. einseitige Druckbelastungen durch appositionelles Wachstum auszugleichen. Im Resultat dessen kommt es zu einem typischen Gestaltswandel bei Kindern und Jugendlichen. Achsen und Torsionen durchlaufen vom Säugling über das Kleinkind zum Erwachsenen charakteristische Veränderungen, wobei sich diese an Ober-, Unterschenkel und Fuß gegenseitig beeinflussen. Während nach Vertikalisierung des Kindes die varische Ausrichtung dominiert (O-Beine), folgt etwa im Alter von 4-8 Jahren eine Phase der leichten Überkorrektur hin zu einem Knievalgus (X-Beine), um schlussendlich nach Verschluss der Wachstumsfugen die physiologische Ausrichtung aufzuweisen. Die mechanische Belastungsachse als wichtigster Indikator für das Alignment der lasttragenden unteren Extremitäten sollte ab diesem Zeitpunkt vom statischen Zentrum der Hüftköpfe durch die Mitte der Kniegelenke in das Zentrum der Sprunggelenke verlaufen (sogenannte Mikulicz Linie). Diese orthograde Ausrichtung der Belastungsachsen ist ein wesentlicher Garant für die verschleißfreie lebenslange Funktion aller Gelenke der unteren Extremitäten.

Um erkennen zu können, ob ein Zustand bei einem Kind pathologisch ist oder noch dem Normbereich zugerechnet werden kann, ist die Kenntnis der normalen Entwicklung der Achsen- und Torsionsverhältnisse von wesentlicher Bedeutung.

Hefti (2000) beschreibt pathologische Formen als selten. Sie kommen kongenital, posttraumatisch, postinfektiös und bei Stoffwechselerkrankungen vor.

Inwieweit und ob Adipositas in den verschiedenen Stadien der kindlichen Entwicklung dazu beiträgt, dass initial physiologische kindliche Achsverhältnisse persistieren oder sich gar progredient hin 
zum Pathologischen verändern, soll Gegenstand der nachfolgenden Betrachtungen sein.

\subsubsection{Tibia vara (Morbus Blount) und Genu varum}

Die idiopathische Tibia vara, auch als Osteochondrosis deformans tibiae oder, nach dem Erstbeschreiber als Morbus Blount (1937) beschrieben, zählt zur Erkrankungsgruppe der aseptischen Osteochondronekrosen. Sie ist durch eine Wachstumsstörung im Bereich der proximalen, medialen Tibiametaphyse und Epiphysenfuge charakterisiert, die zu einer progredienten O-Bein-Deformität (Genu varum) führen kann.

\section{Ätiologie und Pathomechanismus der Tibia vara}

Vermutlich führt die Kombination biologischer und mechanischer Faktoren (Überlastung) zu einer Deformation der proximalen, medialen Tibiaepiphyse (s. Abb. 9a, b). Da sich die Erkrankung gehäuft bei Afroamerikanern findet, wird eine genetisch bedingte Disposition mit in Betracht gezogen. Als prädisponierende Faktoren gelten eine vorbestehende varische Achsausrichtung der betroffenen Extremität in Verbindung mit Übergewicht oder extremer Aktivität und schnellem Wachstum. Auffällig ist eine Persistenz der sich normalerweise im Rahmen des Wachstums zurückentwickelnden physiologischen Genua vara im frühen Kleinkindalter.

Eine andere interessante Hypothese zur Ätiologie der adoleszenten Form der Tibia vara wurde von Davids et al. (1996) präsentiert. In einer biomechanischen Analyse untersuchten sie die These, dass eine erhöhte Belastung des medialen Kniekompartimentes während des Gangzyklus Folge kompensatorischer Gangveränderungen bei vergrößerten Ober-
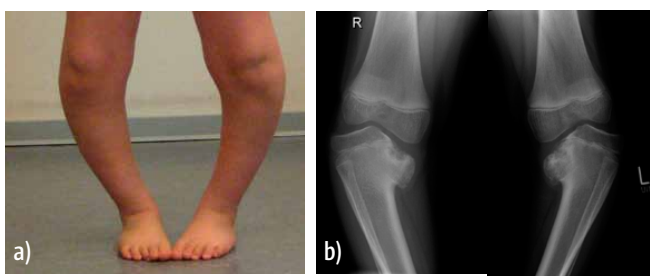

Abb. 9 a, b Ausgeprägte Tibia vara beidseits mit aseptischer Nekrose der medialen Tibiaepiphyse bei einem 6-jährigen Mädchen mit frühmanifester Adipositas, klinisches (a) und radiologisches Bild (b) schenkelumfängen adipöser Individuen sei („fatthigh gait"). Mit Hilfe dreidimensionaler Bewegungsanalysen konnten verschiedene Gangveränderungen wie ein dynamisches Genu varum und eine verstärkte Knierotation in der Standbeinphase sowie eine Zirkumduktion in der Schwungphase identifiziert werden. Diese pathologischen Belastungszyklen können bei den betroffenen Individuen Druckbelastungen erzeugen, die für eine Alteration der Wachstumsfuge ausreichen.

Hauptunterschiede der Tibia vara bestehen im Ausmaß der Deformität und der Rezidivneigung nach erfolgter operativer Korrektur infolge des unterschiedlichen Manifestationsalters.

\subsubsection{Genu valgum}

Bei der Inspektion übergewichtiger Kinder und Erwachsener zeigt sich schnell, dass nicht ausschließlich varische Beinachsen exprimiert werden. Der klinische Aspekt zeigt ebenso häufig eine Valgus(XBein)-Deformität (s. Abb. 10).

Bei der überwiegenden Zahl der unter 10-jährigen Kinder mit einer solchen Deformität handelt es

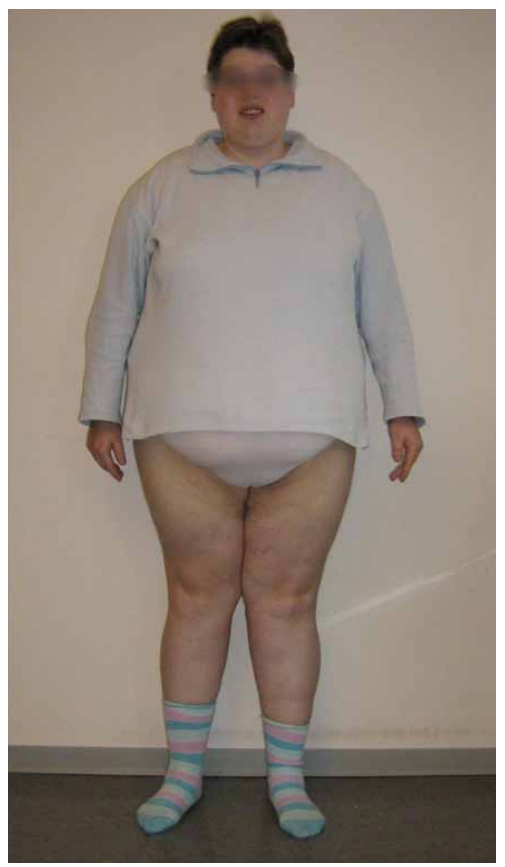

Abb. 10 Symptomatisches Genu valgum (X-Bein) beidseits bei einer 17-jährigen adipösen Jugendlichen 
sich wiederum um Normvarianten im Rahmen der physiologischen Entwicklung der kindlichen Beinachse, die eine spontane Korrektur im weiteren Wachstumsverlauf erfährt. Hefti (2000) schreibt dieser Deformität kaum eine Behandlungsbedürftigkeit $\mathrm{zu}$.

Im Rahmen der Untersuchung muskuloskelettaler Veränderungen bei adipösen Kindern mit Kniebeschwerden beschreiben de Sá Pinto et al. (2006) allerdings gehäuft valgische Beinachsen. Klinisch kann die Entwicklung der Ausprägung der valgischen Beinachse sehr einfach anhand der Messung des Intermalleolarabstandes objektiviert werden. Dabei gelten Werte $>10 \mathrm{~cm}$ als pathologisch.

White et al. (1995) unterstreichen die Notwendigkeit, pathologische Entitäten im Zusammenhang mit fokalen und systemischen Erkrankungen vom physiologischen Verlauf zu unterscheiden. Letztere zeigen eine Progredienz und bedürfen einer Therapie, da bei entsprechender Ausprägung auch das Genu valgum (X-Bein) eine präarthrotische Deformität darstellt.

Die mit dem Übergewicht und der Weichteilzunahme einhergehenden kompensatorischen Veränderungen des Gangzyklus und der Gelenkbiomechanik sind bei Kindern und Jugendlichen wichtige ätiologische Bindeglieder zur Manifestation schwerer wachstumsassoziierter Achsabweichungen der unteren Extremitäten (Tibia vara, Genu varum und valgum).

\subsection{Epiphyseolysis capitis femoris (ECF, kindliches Hüftkopfgleiten)}

Eine besonders schwerwiegende Hüfterkrankung im Kindes- und Jugendalter ist die juvenile Epiphysenlösung der Schenkelhalsepiphysenfuge. Aufgrund der entstehenden Fehlform am Übergang des Hüftkopfes zum Schenkelhals mit hoher Gefahr eines femoroazetabulären Impingement gilt sie als wesentliche Ursache sekundärer Hüftgelenksarthrosen.

\subsubsection{Häufigkeit}

Jungen sind mit 3:1 bevorzugt betroffen. Der Häufigkeitsgipfel der Erkrankung liegt in der Adoleszenz zum Zeitpunkt des größten Wachstumsschubes (Mädchen mit 10-14 Jahren, Jungen mit 11-15 Jahren). Ein beidseitiger Befall, zum Teil zeitlich versetzt, betrifft etwa die Hälfte der Kinder.
Für Japan erfolgte jüngst die Publikation aktueller Daten zur Entwicklung der Inzidenz der ECF im Verlauf der letzten 20-30 Jahre. Noguchi et al. (2002) beschreiben nach Auswertung der Daten einer japanischen Multicenter-Studie eine aktuell vergleichbar hohe Inzidenz der Erkrankung (2,22/100.ooo Jungen und o,76/100.0oo Mädchen) wie sie für die Vereinigten Staaten bereits 1970 angegeben wurde (Kelsey et al. 1970). Er führt dies auf einen vergleichbaren Anstieg adipöser Kinder in Japan zurück. Der Adipositas-Index unter den Patienten dieser Studie ist drei Mal höher als der einer Vergleichsstudie 30 Jahre zuvor (Ninomiya et al. 1976).

Ähnliche Entwicklungen wurden jüngst aus Schottland berichtet, wo die Analyse der Daten eine Steigerung der Inzidenz von 3,78/100.0oo Kindern mit ECF 1981 auf 9,66/100.00o im Jahr 2000 sowie die Senkung des Alters bei Erstmanifestation der Erkrankung zeigte. Parallel dazu konnte durch die Auswertung der Daten der nationalen Vorsorgeprogramme eine stetige Zunahme übergewichtiger Schulkinder in Schottland über die letzten 20 Jahre dokumentiert werden (Murray et al. 2008).

\subsubsection{Pathogenese}

Ein breiter Konsens besteht darin, dass sowohl biomechanische als auch endokrine Faktoren bei der Ätiologie der Erkrankung eine wichtige Rolle spielen. Bei den betroffenen Kindern und Jugendlichen sind sehr häufig konstitutionelle Auffälligkeiten in Form eines Hochwuchses oder mehrheitlich der früher als Fröhlich-Syndrom (Dystrophia adiposogenitalis) beschriebenen Stamm-Adipositas mit Unterentwicklung der Genitalien zu beobachten.

Jingushi et al. (2004) beschreiben die unterschiedlichen Theorien hinsichtlich der Ätiologie der Erkrankung und präsentieren pathogenetische Modelle, welche ausführliche endokrinologische, histomorphologische als auch klinische Daten einbeziehen. Das häufig erhöhte Körpergewicht in Verbindung mit einem während der Pubertät stattfindenden Wachstumsspurt und einer sich dabei verstärkenden Schräglage der Epiphysenfuge (Retrotorsion des Schenkelhalses) gilt als wesentlicher Risikofaktor.

Die mit dem Zeitpunkt des größten longitudinalen Wachstumsschubes einhergehenden temporären hormonellen Veränderungen bei Schilddrüsen-, Wachstums- und Geschlechtshormonen führen zu einer Auflockerung und Erweiterung des kollagenen Netzwerkes der Wachstumsfuge selbst und im Bereich des perichondralen Ringes. Die Resistenz 
gegenüber Scherkräften ist damit vermindert und die Gefahr einer Dislokation der Epiphyse erhöht. Dieser Hypothese folgend sind adipöse Kinder während des pubertären Wachstumsschubes besonders anfällig gegenüber einer Epiphyseolyse. Umso wichtiger erscheint die Sensibilisierung allgemeinärztlich, kinderärztlich und kinderorthopädisch-/chirurgisch tätiger Kollegen gegenüber dieser Erkrankung, um einen beginnenden Abrutsch so früh als möglich zu diagnostizieren und der adäquaten Therapie zuzuführen.

\subsubsection{Klinik und Diagnostik}

Jede Epiphyseolyse führt zu mehr oder weniger stark ausgeprägten Hüft-, Oberschenkel- oder Kniebeschwerden, die leider häufig über längere Zeit verkannt werden.

In der frühen Phase können die Zeichen bei der klinischen Untersuchung sehr unspezifisch sein. Ist der Cleitvorgang fortgeschritten, kommt es zu einem sehr typischen Verlust der Innendrehfähigkeit der betreffenden Hüfte - dies beschreibt das sogenannte Drehmann'sche Zeichen (s. Abb. $11 \mathrm{a}-\mathrm{c}$ ).

Die Diagnose wird meist radiologisch gesichert. Dabei ist zu betonen, dass durch den Abrutsch der Epiphysenfuge nach dorsal häufig auf einer einfachen a.p. Abbildung der betreffenden Hüfte der beginnende Abrutsch „übersehen“ wird. Bei dem klinischen Verdacht auf ein Hüftkopfgleiten muss die betreffende Hüfte immer in zwei Ebenen abgebildet werden. Erst in der zweiten Ebene ist der Abrutsch der Epiphyse klar erkennbar (s. Abb. 12 a, b).

\subsubsection{Therapie}

Die ECF stellt eine kindliche Notfallsituation dar und bedarf immer einer stationären Einweisung, um eine zeitnahe operative Stabilisierung der abgerutschten femoralen Epiphyse vorzunehmen.

\subsubsection{Natürlicher Verlauf}

Fortgeschrittene Cleitvorgänge bergen ein hohes Risiko der Chondrolyse, führen zu einem femoroazetabulären Impingement, Gelenkinkongruenz und starten damit frühzeitig den sekundären Koxarthoseprozess.

Aber auch milde Epiphysenabrutsche führen zu einer Veränderung der Hüftbeweglichkeit und -bio-
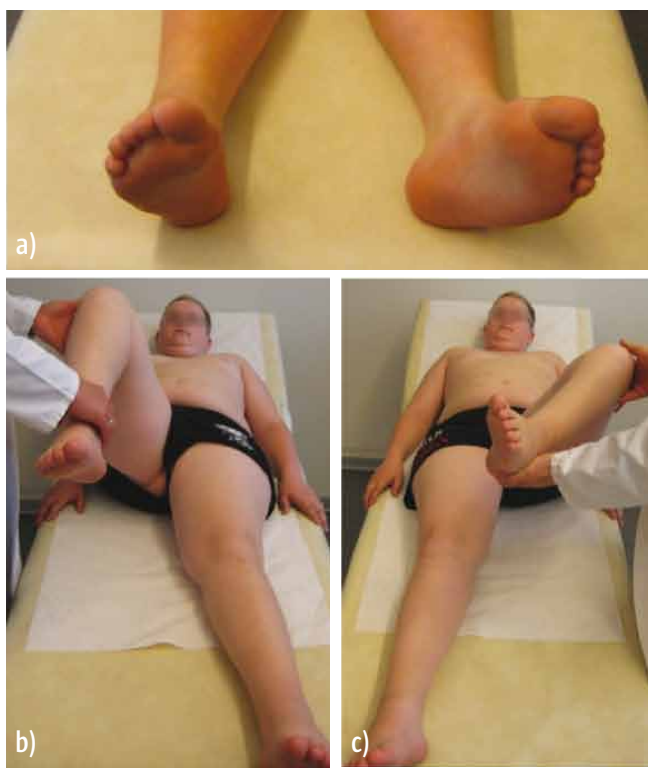

Abb. 11 a-c Typische klinische Befunde bei der Epiphyseolysis capitis femoris: spontane Außendrehfehlstellung des betroffenen Beines (a), und sog. „DrehmannZeichen" als zwanghafte Abduktion und Außendrehung des betroffenen Beines bei passiver oder aktiver Beugung in der Hüfte (b, $c$ )
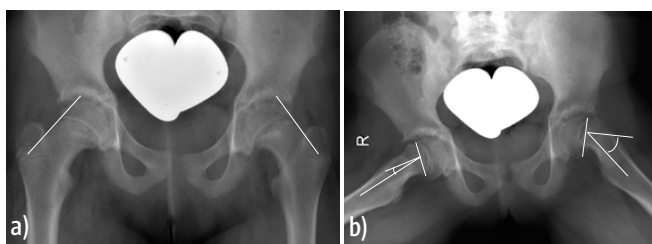

Abb. 12 a, b Die Röntgendiagnostik der Epiphyseolysis capitis femoris erfolgt immer in 2 Ebenen: In der Beckenübersichtsaufnahme (a) ist lediglich eine stärkere Auflockerung der linken Epiphysenfuge erkennbar. Erst in der axialen Abbildung (b) ist der fortgeschrittene Abrutsch der linken Epiphysenfuge und beginnende Abrutsch der rechten Epiphysenfuge nach Dorsal erkennbar; der jeweilige Abrutschwinkel kann vermessen werden.

mechanik. Aufgrund der langen Exposition der daraus resultierenden Fehlform am Femurkopf-Schenkelhalsübergang (sogenannte „tilt“ oder „pistol grip“ Deformität) besteht das Risiko einer Degeneration. Umfangreiche klinische und radiologische Untersuchungen im Rahmen der „UlmerOsteoarthrosestudie“ 
zeigten beispielsweise bei 7,1\% der Studienteilnehmer mit fortgeschrittener Osteoarthrose diese typischen radiologischen Veränderungen der FemurkopfSchenkelhalsform die auf einen mild abgelaufenen Epiphysenabrutsch im Rahmen einer ECF hindeuteten (Günther et al. 1998).

Es kann heute als erwiesen angesehen werden, dass es sich bei der ECF um ein multifaktorielles Geschehen handelt, bei dem der Adipositas ätiologisch zumindest die Rolle eines ganz wesentlichen Kofaktors zukommt. Bei anamnestisch und klinisch nicht klar zuzuordnenden Hüft-oder Kniebeschwerden sollte in der Altersgruppe vor und während der Pubertät immer ein kindliches Hüftkopfgleiten (ECF) durch eine Röntgenaufnahme in zwei Ebenen ausgeschlossen werden. Schwere Verläufe müssen durch eine frühe Sicherung der Diagnose verhindert werden.

\subsection{Adipositas und Arthrose}

Arthrosen großer, lasttragender Gelenke gehören zu den häufigsten Erkrankungen am Bewegungsapparat und haben eine hohe sozialmedizinische Bedeutung (Günther u. Merx 2006). Bei etwa der Hälfte der Betroffenen lassen sich definierte Erkrankungsursachen bzw. Risikofaktoren nachweisen. Als sogenannte „Präarthrosen“ werden Erkrankungen im Kindesalter bezeichnet, die in der Folge schwerwiegende Abweichungen von der anatomisch korrekten Gelenkmorphologie zeigen.

Etwas weniger eindeutig ist die Datenlage zum klinischen und radiologischen Verlauf bei Patienten mit einer anatomisch korrekten Gelenkmorphologie, aber hoher mechanischer Belastung durch eine langjährig bestehende Adipositas. Einem von „Diabetes Australia“ in Auftrag gegebenen Bericht zufolge haben Übergewichtige ein bis zu 2,45-fach erhöhtes Arthrose-Risiko. Epidemiologischen Berechnungen zufolge waren im Jahre 2005 über 225.00o Australier infolge Übergewichts an Arthrose erkrankt - dies entspricht einem Anteil von 14\% aller australischen Arthrosepatienten (Diabetes Australia 2006). In verschiedenen großen Studien (Davis et al. 1990; Felson et al. 1992) wurde bereits vor 20 Jahren auf den $\mathrm{Zu}-$ sammenhang zwischen Übergewicht und der Entwicklung der Kniearthrose hingewiesen. Felson et al. (1992) beschreiben für das von ihnen untersuchte weibliche Kollektiv gleichfalls die Senkung der Inzidenz behandlungsbedürftiger Kniearthrosen allein durch die Gewichtsreduktion.
Im Rahmen der Ulmer Osteoarthrosestudie wurden durch Stürmer et al. (2000) auch Zusammenhänge zwischen einer bestehenden Adipositas bzw. Übergewichtigkeit und radiologischen Arthrosemerkmalen bei Männern und Frauen mit fortgeschrittener Knie- und Hüftarthrose untersucht. In Bezug auf eine radiologisch fortgeschrittene Kniearthrose konnte ein solcher Zusammenhang mit Adipositas bzw. Übergewicht bestätigt werden. Dieser wurde noch deutlicher bei zusätzlicher Achsabweichung der Extremität. Kein Zusammenhang konnte jedoch zwischen Körpergewicht und bilateraler Arthrose der Hüften gefunden werden.

\section{Einfluss von Übergewicht auf das Ergebnis nach endoprothetischem Hüft- und Kniegelenksersatz}

Beim Vorliegen einer fortgeschrittenen, mit konservativer Behandlung nicht beherrschbaren Arthroseerkrankung ist in der Regel der künstliche Gelenkersatz indiziert. Das Hauptziel ist hierbei die Verbesserung der Lebensqualität von Patienten mit fortgeschrittenen Gelenkbeschwerden durch Schmerzlinderung und Verbesserung der Gelenkfunktion. Aufgrund der zunehmend verbesserten Prothesenstandzeiten, hoher Patientenzufriedenheit und Kosteneffektivität (Katz 20o1) hat die Gesamtzahl an endoprothetischen Hüft- und Kniegelenkseingriffen in den vergangenen Jahren weltweit zugenommen.

Der mögliche Einfluss von Adipositas auf das Ergebnis nach endoprothetischer Versorgung wird aktuell noch sehr kontrovers diskutiert. Im Rahmen einer internationalen Multicenter-Querschnittsuntersuchung der EUROHIP Forschergruppe konnte ein Mangel an Konsensus bezüglich des Einflusses von Übergewicht auf das Ergebnis nach hüftendoprothetischer Versorgung zwischen Ärzten unterschiedlicher Fachgebiete aufgedeckt werden. So waren einweisende Ärzte in geringerem Maße als orthopädisch operativ tätige Kollegen der Meinung, Adipositas gehe mit einem erfolgreichen Ergebnis nach hüftendoprothetischer Versorgung einher (Stürmer et al. 2005). Einerseits argumentieren verschiedene Autoren, dass übergewichtige bzw. adipöse Patienten neben erhöhten Raten allgemeiner ( $z$. B. Thromboembolie) und spezieller (z.B. Blutung, Infektion, Prothesenlockerung, periprothetische Fraktur) Komplikationen einen geringeren Nutzen im Rahmen einer hüftendoprothetischen Versorgung im Vergleich zu normalgewichtigen Patienten zu erwarten hätten (Namba et al. 2005). Andererseits existieren Hinweise darauf, dass bei adipösen Patienten keines- 
falls mit schlechteren langfristigen Ergebnissen nach Hüft-TEP Implantation zu rechnen sei (Andrew et al. 2008; McLaughlin et al. 2006). Eine eigene prospektive Nachuntersuchung an einem größeren $\mathrm{Pa}$ tientenkollektiv im Rahmen des Dresdner Hüftregisters (unveröffentlichte Daten) deutet darauf hin, dass das Vorliegen von Übergewicht (BMI 25-30) oder Adipositas (BMI > 30) keinen nachteiligen Effekt auf die Gelenkfunktion und Lebensqualität kurz- bis mittelfristig nach erfolgter Hüftendoprothesenimplantation aufweist.

Die Bedeutung, die lokalen biomechanischen und systemisch-metabolischen Faktoren bei der Entstehung von Hüftgelenksarthrose in einer adipösen Population zukommt, wird kontrovers diskutiert. Für die Entstehung einer Kniegelenksarthrose gilt der Zusammenhang zu einem erhöhten individuellen BMI als gesichert.

Die Frage, ob adipöse Patienten weniger als Normalgewichtige von einer endoprothetischen Versorgung profitieren, kann unter Berücksichtigung der aktuell vorliegenden wissenschaftlichen Literatur noch nicht abschließend beantwortet werden.

\subsection{Rückenschmerz}

Schmerzen im Bereich des Achsenskeletts betreffen häufig die lumbalen Segmente und sind meist Folge einer Dysfunktion oder Degeneration der Bandscheiben oder der kleinen Wirbelgelenke mit konsekutiven muskulo-ligamentären Tonusveränderungen. Im fortgeschritten Stadium der Degeneration können durch protrahierte Bandscheibenanteile, segmentale Instabilitäten oder degenerativ hypertrophierte Kapselbandstrukturen neuronale Strukturen kompromittiert werden, was bis hin zu sensomotorischen Ausfallserscheinungen führen kann.

Während für Erwachsene und ältere Menschen Übergewicht und Adipositas als ein wichtiger Risikofaktor für die Degeneration der Bandscheiben und für das Auftreten von Rückenschmerz identifiziert werden konnte (Hangai et al. 2007; Shiri et al. 2008) existieren nur wenige Untersuchungen, die auf den Zusammenhang von Wirbelsäulenschmerz und Stammfettsucht bei Kindern und Jugendlichen eingehen. Schmerzen gelten allgemein als ein Frühsymptom drohender degenerativer Veränderungen. Insofern gilt es, identifizierte Risikofaktoren einer Bandscheibendegeneration bei Erwachsenen auch

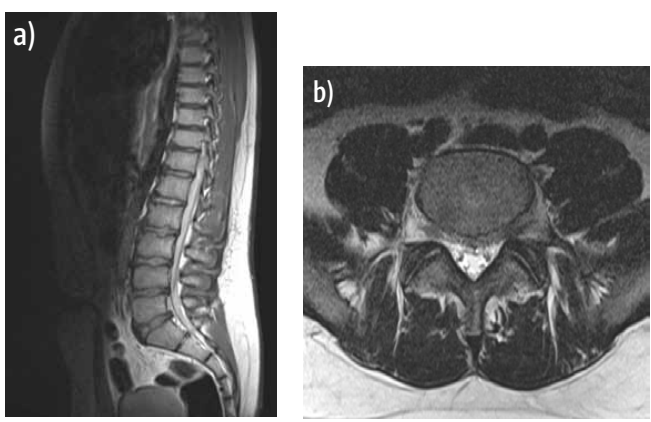

Abb. 13 a, b Altersuntypische polysegmentale lumbale Veränderungen im MRT bei einem übergewichtigen 14-jährigen Jungen (BMI 30,4), der aufgrund akuter Schmerzen mit neurologischen Ausfällen im Sinne einer Claudicatio spinalis stationär zugewiesen wurde.

bei Wirbelsäulenbeschwerden im Kindes- und Jugendalter ernstzunehmen und einer Behandlung zuzuführen. Stovitz et al. (2008) beschreiben in einer jüngst veröffentlichten Feldstudie mit 135 adipösen Kindern und Jugendlichen, die sich im Rahmen einer pädiatrischen Behandlung einer Adipositasberatung unterzogen, dass die Mehrzahl dieser Kinder (61\%) einmal pro Monat Schmerzen in mindestens einem Gelenk angeben. Mit 39\% war die Prävalenz von Rückenbeschwerden in dieser Population, gefolgt von Knie- $(24 \%)$, Sprunggelenks- $(15 \%)$ und Fußbeschwerden (26\%) am höchsten. Ähnliche Daten werden von Taylor et al. (2006) und Bell et al. (2007) berichtet.

Altersuntypische frühzeitige Wirbelsäulenveränderungen einschließlich neurologischer Ausfälle konnten bei Jugendlichen mit starkem Übergewicht auch im eigenen Patientengut vereinzelt beobachtet werden (s. Abb. 13 a, b).

Die gehäufte Angabe von Wirbelsäulenbeschwerden bei adipösen Kindern- und Jugendlichen lässt eine Koinzidenz bei der Entstehung degenerativer Veränderungen am Achsenskelett im Erwachsenenalter vermuten. Für erwachsene Patienten konnte der Nachweis dieser Koinzidenz zwischen degenerativen Veränderungen der Wirbelsäule und Übergewicht bereits geführt werden. 


\section{Fazit}

Mit der Verbesserung von Lebensstandards und der immer leichteren Verfügbarkeit von Nahrungsmitteln wird seit vielen Jahren in den Industriestaaten eine parallele Zunahme von übergewichtigen Menschen beobachtet. Diese Tatsache führte neben dem Anstieg von kardiovaskulären und Stoffwechselerkrankungen auch zum gehäuften Auftreten von Beschwerden am muskuloskelettalen Apparat. Die aus den Beschwerden resultierende Inaktivität scheint für viele der Betroffenen einen Teufelskreislauf zu unterhalten, den es durch Aufklärung und Aufzeigen von Alternativen frühzeitig zu durchbrechen gilt. Insbesondere für die degenerativ-arthrotischen Veränderungen an den lumbalen Wirbelsäulensegmenten und am Kniegelenk konnten wissenschaftlich fundierte Zusammenhänge zu Übergewicht und Adipositas aufgezeigt werden.

Mit einem schlecht eingestellten Diabetes mellitus steigt, je nach zeitlicher Länge der Exposition, die Zahl diabetischer Fußprobleme. Das diabetische Fußsyndrom wird oftmals unterschätzt und bedarf einer interdisziplinär geführten Betreuung. Die bestmögliche Einstellung der Stoffwechselveränderungen, Beratungs- und Schulungsmaßnahmen, die richtige Wundbehandlung und orthopädieschuhtechnische Anpassungen können oft Schlimmstes verhindern helfen.

Leider werden Übergewicht, Adipositas und deren Folgen nicht nur bei erwachsenen Individuen sondern zunehmend auch bei Kindern und Adoleszenten manifest. Insbesondere am wachsenden Skelett sind Übergewicht und Adipositas mit schweren strukturellen Störungen der Wachstumsfugen sowie mit sekundären Form- und Achsabweichungen an den lasttragenden unteren Extremitäten assoziiert. Diese Achsabweichungen stellen die betroffenen Kinder und Jugendlichen nicht nur vor kosmetische und funktionelle Probleme, sondern potenzieren auch das spätere Risiko eines degenerativen Gelenkverschleißes. Fachgruppen die mit der Betreuung übergewichtiger Kinder und Jugendlicher betraut sind, sollten die im Rahmen bestimmter Entwicklungsphasen gehäuft auftretenden Erkrankungen am Skelettsystem kennen und auf Frühsymptome besonders sensibel reagieren.

Dieser Beitrag ist eine aktualisierte und gekürzte Fassung der Originalpublikation: Thielemann F, Günther KP, Stiehler M. Einfluss von Übergewicht und Adipositas auf den wachsenden und adulten Bewegungsapparat. Adipositas 2010; 4: 26-35. Mit freundlicher Genehmigung der Schattauer $\mathrm{GmbH}$.

\section{Literatur}

Andrew JG, Palan J, Kurup HV, Gibson P, Murray DW, Beard DI (2008) Obesity in total hip replacement. J Bone Joint Surg Br 90, 424-9

Bell LM, Byrne S, Thompson A, Ratnam N, Blair E, Bulsara M, Jones TW, Davis EA (2007) Increasing body mass index z-score is continuously associated with complications of overweight in children, even in the healthy weight range. I Clin Endocrinol Metab 92, 517-22

Blount WP (1937) Tibia vara: osteochondrosis deformans tibiae. | Bone Joint Surg 19A, 1-8

Bruckner L, Rosler P (1981) Orthopaedic findings of the foot as related to age and body weight among women. ZFA 36, 29-35

Davids IR, Huskamp M, Bagley AM (1996) A Dynamic Biomechanical Analysis of the Etiology of Adolescent Tibia Vara. J Pediatr Orthop 16, 461-468

Davis MA, Ettinger WH, Neuhaus IM (1990) Obesity and osteoarthritis of the knee: evidence from the national health and nutrition examination survey (NHANES I). Semin Arthritis Rheum 20, 34-41

Diabetes Australia (2006) The Economic Costs of Obesity. Canberra: Access-Economics

Dowling AM, Steel JR, Baur LA (2001) Does obesity influence foot structure and plantar pressure patterns in prepubescent children. Int I Obes Relat Metab Disord 25, 845-52

Dowling AM, Steel JR, Baur LA (2004) What are the effects of obesity in children on plantar pressure distribution? International Journal of Obesity 28, 1514-1519

Felson DT, Zhang Y, Anthony JM, Naimark A, Anderson II (1992) Weight loss reduces the risk for symtomatic knee osteoarthritis in women. Ann Intern Med 116, 535-539

Günther KP, Stürmer T, Sauerland S, Zeissig I, Sun Y, Kessler S, Scharf HP, Brenner H, Puhl W (1998) Prevalence of generalized osteoarthritis in patients with advanced hip and knee OA: the Ulm osteoarthritis Study. Ann Rheum Dis 57, 717-723

Günther KP, Merx H (2006) Epidemiologie und sozialmedizinische Bedeutung der Arthrose. In: J. Zacher Hrsg) Herausforderung Arthrose. Berufsverband der Fachärzte für Orthopädie e.V.

Hangai M, Kaneoka K, Kuno S, Hinotsu S, Sakane M, Mamizuka N, Sakane M, Ochiai N (2007) Factors associated with lumbar intervertebral disc degeneration in the elderly. Spine I Nov 21

Hefti F (2000) Achsenfehler an den unteren Extremitäten. Orthopäde 29, 814-20

Horn MR (2002) Orthopädische Komorbidität bei Kindern und Jugendlichen mit Adipositas unter besonderer Berücksichtigung der Epiphysiolysis capitis femoris. Med. Promotion, Universität Ulm

Jingushi S, Suenaga E (2004) Slipped capital femoral epiphysis: etiology and traetment. / Orthop Sci 9, 214-219

Katz IN (2001) Preferences, quality, and the (under)utilization of total joint arthroplasty. Med Care 39, 203-5

Kelsey JL, Keggi KI, Southwick WO (1970) The incidence and distribution of slipped femoral epiphysis in Connecticut and southwestern United States. J Bone Joint Surg 52A, 1203-1216

McLaughlin JR, Lee KR (2006) The outcome of total hip replacement in obese and non-obese patients at 10-to 18-years. I Bone Joint Surg Br 88, 1286-92 
Mertz DP (1982) Hallux rigidus arthrosis and gout. Fortschr Med 100, 446-8

Murray AW, Wilson NIL (2008) Changing incidence of slipped capital femoral epiphysis. A relationship with obesity? J Bone Joint Surg (Br) 90-B, 92-4

Namba RS, Paxton L, Fithian DC, Stone ML (2005) Obesity and perioperative morbidity in total hip and total knee arthroplasty patients. I Arthroplasty 20 (7 Suppl 3), 46-50

Ninomiya S, Nagasaka Y, Tagawa H (1976) Slipped capital femoral epiphysis: a study of 68 cases in the eastern half area of Japan. Clin Orthop 119, 172-6

Noguchi Y, Sakamaki T (2002) The Multicenter Study Committee of the Japanese Pediatric Orthopaedic Association. Epidemiology and demographic of slipped capital femoral epiphysis in Japan: a multi-center study by the Japanese Pediatric Orthopaedic Association. J Orthop Sci 7, 610-17

Riddiford-Harland DL, Steel JR, Storlien LH (2000) Does obesity influence foot structure in prepubescent children? Int I Obes Relat Metab Disord 24, 541-4

de Sá Pinto AL, de Barros Holanda PM, Radu AS, Villares SM, Lima FR (2006) Musculoskeletal findings in obese children. I Peadiatr Child Health 42, 341-4
Shiri R, Solovieva S, Husgafvel-Pursiainen K, Taimela S, Saarikoski LA, Huupponen R, Viikari I, Raitakari OT, Viikari-Juntura E (2008) The association between obesity and the prevalence of low back pain in young adults: the Cardiovascular Risk in Young Finns Study. Am I Epidemiol 167, 1110-9

Stovitz SD, Pardee PE, Vazquez G, Duval S, Schwimmer JB (2008) Musculoskeletal pain in obese children and adolescents. Acta Paediatr 97, 489-93

Stürmer T, Günther KP, Brenner H (2000) Obesity, overweight and patterns of osteoarthritis: the Ulm Osteoarthritis Study. I Clin Epidemiol 53, 307-313

Stürmer T, Dreinhöfer K, Gröber-Grätz D, Brenner H, Dieppe P, Puhl W, Günther KP (2005) Differences in the views of orthopaedic surgeons and referring practitioners on the determinants of outcome after total hip replacement. | Bone Joint Surg $\mathrm{Br} 87,1416-9$

Taylor ED, Theim KR, Mirch MC, Ghorbani S, Tanofsky-Kraff M, AdlerWailes DC, Brady S, Reynolds IC, Calis KA, Yanovski JA (2006) Orthopedic complications of overweight in children and adolescents. Pediatrics 117, 2167-74

White GR, Mencio GA (1995) Genu valgum in children: Diagnostic and therapeutic alternatives. I Am Acad Orthop Surg 3, 275-83

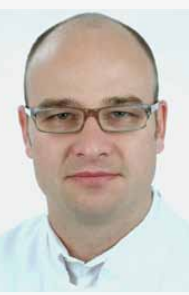

\section{Dr. med. Falk Thielemann}

Nach dem Studium der Humanmedizin an der Charité der Humboldt Universität Berlin und den Medizinischen Fakultäten der TU Dresden, der Universität Marseille, Genf und Toronto folgte die chirurgische Ausbildung als Assistenzarzt am Krankenhaus Pirna und die orthopädische Ausbildung als wissenschaftlicher Mitarbeiter am Universitätsklinikum Dresden. Hier promovierte er 1995 und erlangte 2001 den Facharzt für Orthopädie, 2006 die Zusatzbezeichnung Kinder-Orthopädie und 2007 den Facharzt für Orthopädie und Unfallchirurgie. Der klinische und wissenschaftliche Schwerpunkt seiner Tätigkeit liegt im Bereich der Kinderorthopädie und rekonstruktiven Extremitätenchirurgie. Die Sektion Hand, Fuß und Kinderorthopädie der Orthopädischen Klinik am Universitätsklinikum Dresden wird von inm geleitet. Er ist Mitglied der Deutschen Gesellschaft für Orthopädie und Unfallchirurgie (DGOU) und der deutschen Vereinigung für Kinderorthopädie (VKO). 


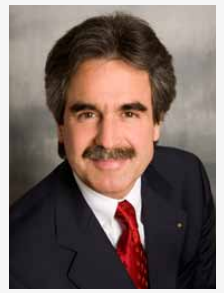

\section{Prof. Dr. med. Klaus-Peter Günther}

Prof. Günther studierte Humanmedizin an der Ludwig-Maximilians-Universität München sowie an den Universitäten von San Francisco und Los Angeles. Es folgte die Assistenzarztzeit am Krankenhaus Traunstein, am Uniklinikum Zürich und später an der Universität Ulm, wo er 1992 seinen Facharzt erlangte und 1997 habilitiert wurde. 2002 folgte der Ruf an die Medizinische Fakultät der TU Dresden, wo Prof. Günther seither den Lehrstuhl für Orthopädie innehat und als Ärztlicher Direktor die Klinik und Poliklinik für Orthopädie leitet.

Seit 2003 ist Prof. Günther Vorstandsmitglied der Europäischen Orthopädenvereinigung (EFORT) und seit 2004 Mitglied im Steering Committee der OMERACT/OARSI-Arbeitsgruppe „Outcome measures in osteoarthritis“. 2009 übernahm Prof. Günther für ein lahr die Präsidentschaft der Deutschen Gesellschaft für Orthopädie und Orthopädische Chirurgie (DGOOC). Aktuell ist er Präsident der Arbeitsgemeinschaft Endoprothetik (AE) für die Jahre 2010 bis 2012. Sein Forschungsschwerpunkt liegt in der Epidemiologie und Versorgungsforschung degenerativer Gelenkerkrankungen sowie im Bereich der rekonstruktiven Hüftchirurgie.

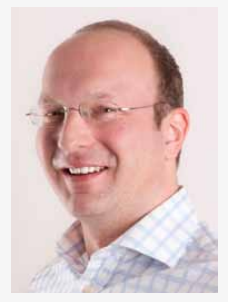

\section{Dr. med. Maik Stiehler, Ph.D.}

Nach dem Medizinstudium an der Georg-August-Universität Göttingen, Harvard Medical School Boston, MA, USA und Universität Bern, Schweiz arbeitete Dr. Stiehler am anatomischen Institut der Universität Göttingen. 2002 bis 2006 war er wissenschaftlicher Mitarbeiter und Assistenzarzt an der Orthopädischen Klinik, Universitätsklinikum Aarhus, Dänemark. 2006 Promotion (Dr. med.) auf dem Gebiet der Nuklearmedizin, Philipps-Universität Marburg und Erlangung des Ph.D.-Grades auf dem Gebiet der regenerativen Knochenforschung, Universität Aarhus. 2006 bis 2010 Assistenzarzt Orthopädische Klinik, Universitätsklinikum Carl Gustav Carus Dresden, seit 2009 dort Leiter der Experimentellen Forschungsgruppe und Knochenbank sowie Leiter der deutsch-chinesischen Juniornachwuchswissenschaftlergruppe „Moderne Anwendungen der Biotechnologie“. Seit 2010 Facharzt für Orthopädie und Unfallchirurgie mit Schwerpunkt Endoprothetik und Hüftchirurgie. 